\title{
Spectrogram Image Analysis of Error Signals for Minimizing Impulse Noise
}

\author{
Jeakwan Kim, Yunseon Choi, and Young-Sup Lee \\ Department of Embedded Systems Engineering, University of Incheon, 119 Academy-ro, Yeonsu-gu, Incheon 406-772, Republic of Korea \\ Correspondence should be addressed to Young-Sup Lee; ysl@incheon.ac.kr
}

Received 16 March 2015; Accepted 8 July 2015

Academic Editor: Marco Anisetti

Copyright ( 2016 Jeakwan Kim et al. This is an open access article distributed under the Creative Commons Attribution License, which permits unrestricted use, distribution, and reproduction in any medium, provided the original work is properly cited.

\begin{abstract}
This paper presents the theoretical and experimental study on the spectrogram image analysis of error signals for minimizing the impulse input noises in the active suppression of noise. Impulse inputs of some specific wave patterns as primary noises to a onedimensional duct with the length of $1800 \mathrm{~mm}$ are shown. The convergence speed of the adaptive feedforward algorithm based on the least mean square approach was controlled by a normalized step size which was incorporated into the algorithm. The variations of the step size govern the stability as well as the convergence speed. Because of this reason, a normalized step size is introduced as a new method for the control of impulse noise. The spectrogram images which indicate the degree of the attenuation of the impulse input noises are considered to represent the attenuation with the new method. The algorithm is extensively investigated in both simulation and real-time control experiment. It is demonstrated that the suggested algorithm worked with a nice stability and performance against impulse noises. The results in this study can be used for practical active noise control systems.
\end{abstract}

\section{Introduction}

Active noise control (ANC) systems are usually seeking to maximize the attenuation of a primary noise by cancelling the unwanted noise by taking advantage of the principle of superposition $[1,2]$. They use adaptive feedforward algorithms such as the filtered reference least mean square (FxLMS) to compensate for the effect of the secondary path in order to ensure convergence $[1,2]$. This ANC has been widely applied successfully to a number of applications such as airplanes, cars, headsets, mobile devices, and other consumer electronics $[3,4]$.

However, as it is necessary to suppress a sudden impulse noise, an advanced ANC system, therefore, needs to have a capability to attenuate the sudden impulse noise to a certain level [5-8]. The spectrogram images give good information on how much the impulse input noise is suppressed after the application of the ANC algorithm [9-15].

Impulse noises are important but harmful sources of the input to a number of practical control systems in which the noise level needs to maintain a certain level such as passenger cars or other various vehicles. Passenger cars are frequently exposed to excessive impulse noises when they are driven on rough roads or various bumps on pavements. Sudden impulses give huge effects to active noise control systems in terms of control stability as they can invoke excessive responses to maintain the performance during control. Therefore, it is necessary to adapt the step size to the magnitude of the impulses to control it, and the FxNLMS (filtered-x normalized LMS) algorithm can be used suitably in this case. For better driving and travelling conditions for drivers and passengers, they need to keep a comfortable noise level. The FxNLMS can offer better calm environment rather than FxLMS algorithm especially against impulse noise inputs. In this study, the algorithm for a duct ANC system is investigated in depth in order to control some impulse signals made of specific half-sine waves.

In this study, thus, the analysis of the spectrogram images of error signals before and after control against three different impulses input noises to a duct system is shown.

The rest of the paper is organized as follows. In Section 2, theoretical considerations are carried out to derive proper equations for impulse noise control based on the FxNLMS algorithm with a normalization factor in a duct. Section 3 presents the experimental setup including the test duct and control board for the real-time control. Also, the modelling 


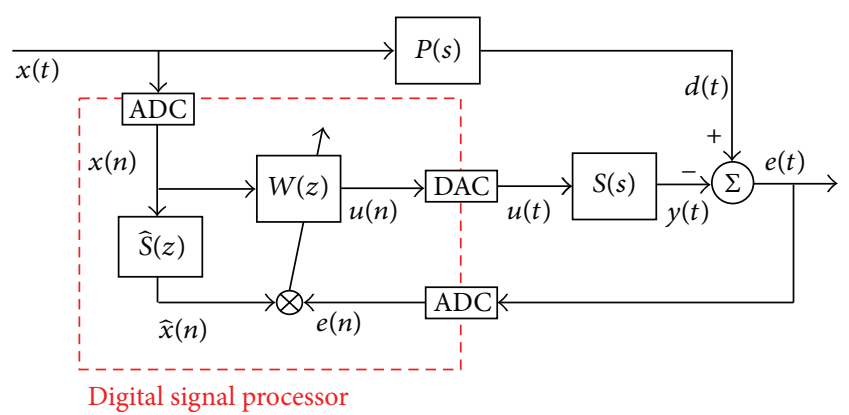

FIgURE 1: FXLMS algorithm.

of the secondary path is achieved to implement it in the control algorithm and the impulse signals for the input signal are designated in the same section. Intensive analysis and discussions from the measured results from real-time control in terms of time and frequency domain are written in Section 4. Finally, conclusions are summarized in Section 5.

\section{Control Algorithm for Minimizing Impulse Noises}

An impulse input to a system to be controlled causes a sudden response which makes the normal FxLMS cannot work properly. As shown in Figure 1, the FxLMS update equation of the adaptive filter $W(z)$ is expressed as

$$
\mathbf{w}_{n+1}=\mathbf{w}_{n}+\alpha e \widehat{\mathbf{x}},
$$

where $\alpha$ is the convergence coefficient and $e$ is the error signal and $\widehat{\mathbf{x}}$ is the filtered reference signal vector which passed through the secondary path model $\widehat{S}(z)$ as illustrated in Figure 1.

Also, as the large impulse input signal can introduce the instability in the LMS algorithm, one can use a smaller convergence coefficient when the measured reference signal becomes suddenly huge. This can lead to a stable FxLMS algorithm by modifying the step size of the update of the adaptive filter $W(z)$ by replacing $\alpha$ in (1) with $\alpha_{N}$ which can be defined by

$$
\alpha_{N}=\frac{\alpha}{\widehat{\mathbf{x}}^{T}(n) \widehat{\mathbf{x}}(n)}
$$

In (2), $\alpha_{N}$ becomes smaller when the filtered reference signal $\widehat{\mathbf{x}}$ is suddenly large. This is known as the FxNLMS (filtered-x normalized LMS). However, when the interval of each impulse is small enough, the system will respond continuously before the previous response decays completely. Thus, a new suggestion for the convergence coefficient can be written as

$$
\alpha_{N C}=k \frac{\alpha}{\widehat{\mathbf{x}}^{T}(n) \widehat{\mathbf{x}}(n)},
$$

where the new term $k$ increases the step size. Thus, $\alpha_{N C}$ takes the place of $\alpha$ in (1) and it is rewritten as

$$
\mathbf{w}_{n+1}=\mathbf{w}_{n}+\alpha_{N C} e \widehat{\mathbf{x}}
$$

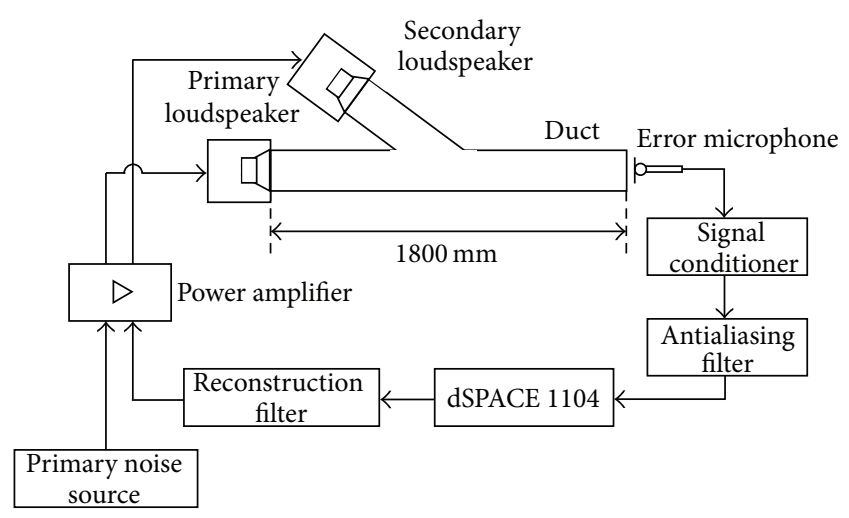

FIgURE 2: Experiment setup for the real-time control.

TABLE 1: The specifications of the components in the duct system.

\begin{tabular}{lc}
\hline Component & Specification \\
\hline Duct & Acryl, 1800 mm \\
Loudspeakers & Sammi SR-100A50 \\
Microphone & PCB 1/2" 377B11 \\
Signal conditioner & PCB 442B104 \\
Antialiasing filter, & Low-pass filter, \\
reconstruction filter & cutoff frequency $=500 \mathrm{~Hz}$ \\
Digital signal processor & dSPACE DS1104 \\
Power amplifier & InterM QD4240 \\
\hline
\end{tabular}

Based on the suggested control algorithm in (4), the active minimization of the impulse input noise in a duct system will be presented in Section 3 in detail. As the algorithm can adaptively follow the huge and sudden changes in the input signal as denoted $x(t)$ in Figure 1, the control experiments were performed to demonstrate the feasibility of suppressing the impulse input noises in terms of the spectrogram images.

\section{Experimental Setup}

3.1. Hardware Setup. For the real-time impulse noise control, a duct experimental system has been built. The system includes a dSPACE 1104 digital signal processor, an acrylic duct, a primary loudspeaker at the end, a secondary loudspeaker, an error microphone at the other side end, a power amplifier, a signal conditioner, a reconstruction filter, and an antialiasing filter as shown in Figure 2. The distance between the primary loudspeaker and the error microphone is $1800 \mathrm{~mm}$.

The primary noise was originated from an external source and the control signal was generated from dSPACE 1104. The sampling frequency for the experiment was $6,000 \mathrm{~Hz}$. The reconstruction filter and the antialiasing filter were designed as low-pass filters and their cutoff frequencies were both $500 \mathrm{~Hz}$. Table 1 shows the specifications of the components used in the experimental duct system in detail. In the realtime control experiment, the fixed length of the signal $\widehat{\mathbf{x}}$ in the denominator of (2) for the FxNLMS was 100. 

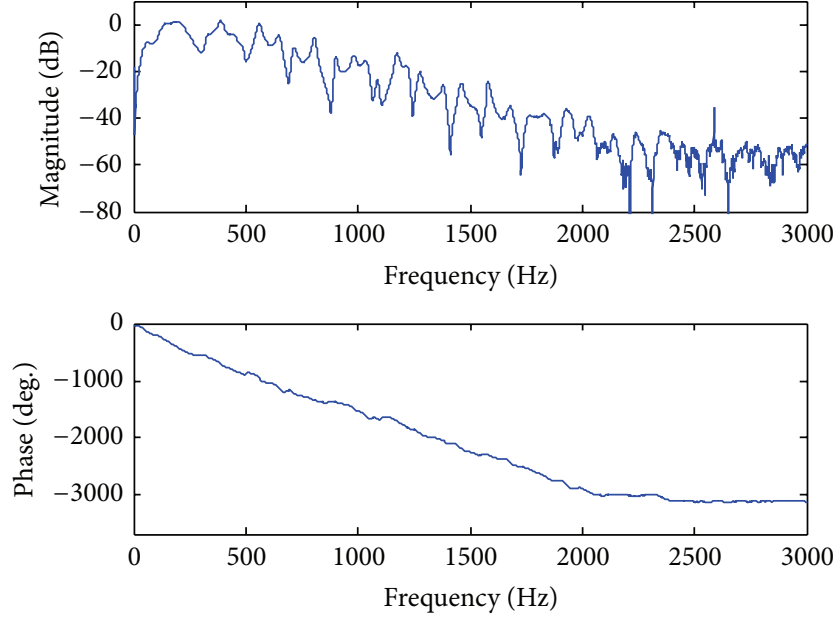

(a)

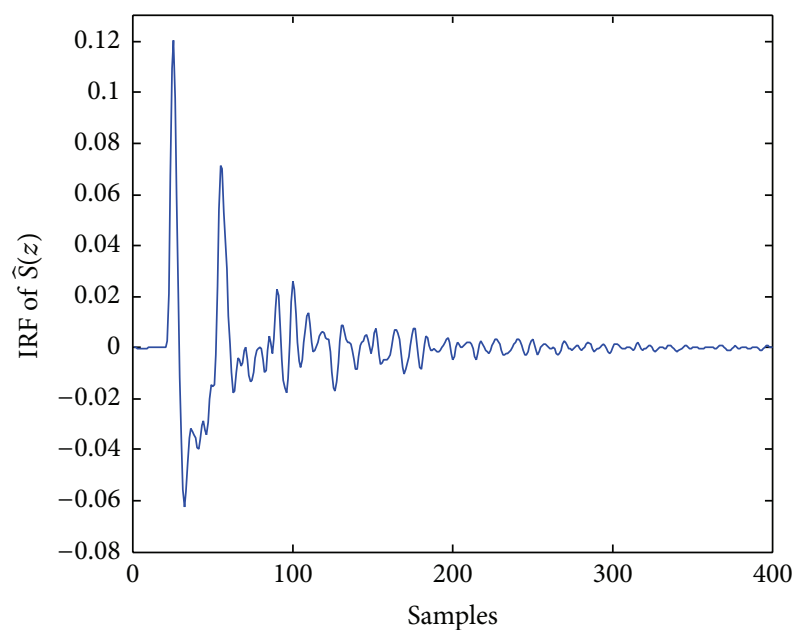

(b)

FIGURE 3: Responses of the secondary path. (a) FRF, (b) IRF.

3.2. Plant Modelling. The modeling of the secondary path of the duct system was carried out for the implementation of the control system. Its frequency response function (FRF) and impulse response function (IRF) are plotted in Figures 3(a) and $3(\mathrm{~b})$, respectively.

The FRF indicates that there are many modes in the plant and the phase shows a linear property between 0 and about $2,000 \mathrm{~Hz}$ although there are some sudden changes at certain frequencies such as about $500 \mathrm{~Hz}$ and $1,100 \mathrm{~Hz}$ as illustrated in Figure 3(a).

The length of the IRF reaches about 400 samples until the response is decayed enough when the sampling frequency is $6,000 \mathrm{~Hz}$ as plotted in Figure 3(b). However, the IRF length to represent the secondary path $\widehat{S}(z)$ was just 50 samples, because of the complexity of the control algorithm applied in this experiment.

3.3. Impulse Input Signals. The impulse inputs signals were designed by combining with half-sine waves and white noises to test the stability and performance of the control algorithm against some sudden impulse noises as described in Section 2. As shown in Figure 4, the three half-sine waves with the frequencies of $77 \mathrm{~Hz}, 209 \mathrm{~Hz}$, and $431 \mathrm{~Hz}$, respectively, were designed for the control experiment. The sampling frequency of the control experiment was $6,000 \mathrm{~Hz}$, and this indicates that 39 samples were necessary to implement the $77 \mathrm{~Hz}$ halfsine wave in Figure 4. And 15 and 7 samples were used for $209 \mathrm{~Hz}$ and $431 \mathrm{~Hz}$ half-sine waves, respectively.

Actually, the three different patterns of the half-sine waves represent some typical impulse noises. For example, the drivers of passenger cars can experience sudden big impulse noises when they pass over bumps on various roads. There are many different bumps on roads in terms of their heights and lengths. The three half-sine waves of 77,209 , and $431 \mathrm{~Hz}$ indicate blunt, medium, and sharp bumps, respectively. White noises were combined in the half-sine waves to make the impulse input signals contain more realistic background disturbances.

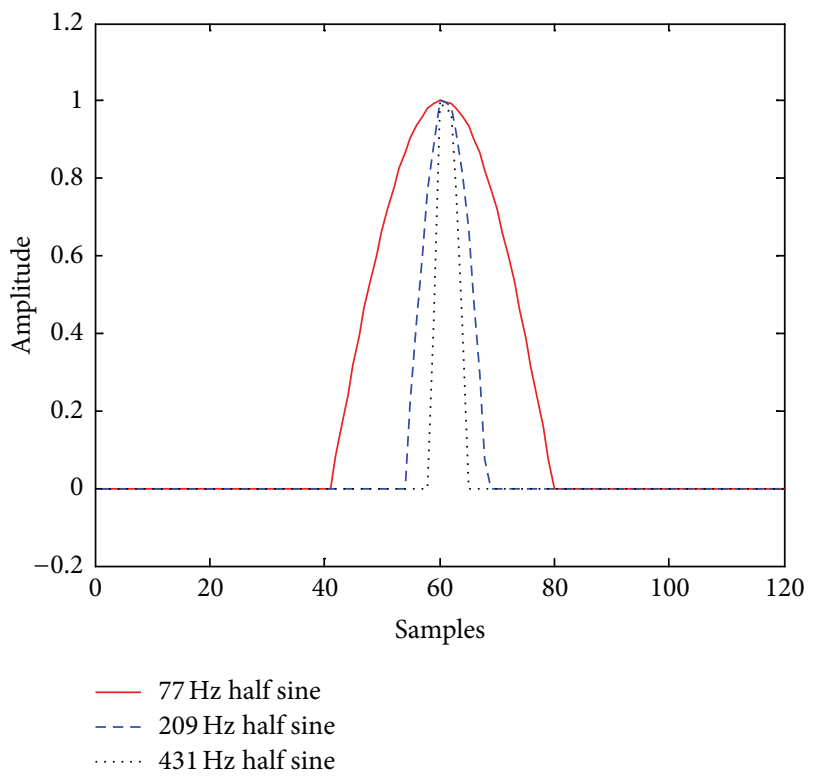

FIGURE 4: Half-sine waves of $77 \mathrm{~Hz}, 209 \mathrm{~Hz}$, and $431 \mathrm{~Hz}$ for impulse input signals.

TABLE 2: The specifications of the components in the duct system.

\begin{tabular}{lc}
\hline $\begin{array}{l}\text { Impulse input } \\
\text { type }\end{array}$ & Signal combination \\
\hline A & 14 of $77 \mathrm{~Hz}$ half-sine waves + white noise \\
B & 14 of $209 \mathrm{~Hz}$ half-sine waves + white noise \\
C & 5 of $77 \mathrm{~Hz}+5$ of $209 \mathrm{~Hz}+4$ of $431 \mathrm{~Hz}$ \\
& half-sine waves + white noise \\
\hline
\end{tabular}

Then, three impulse inputs of types $\mathrm{A}, \mathrm{B}$, and $\mathrm{C}$ were considered as shown in Table 2. Type A impulse input consists of randomly spaced 14 impulses of $77 \mathrm{~Hz}$ half-sine wave with white noise for 2 seconds. Type B input has randomly spaced 


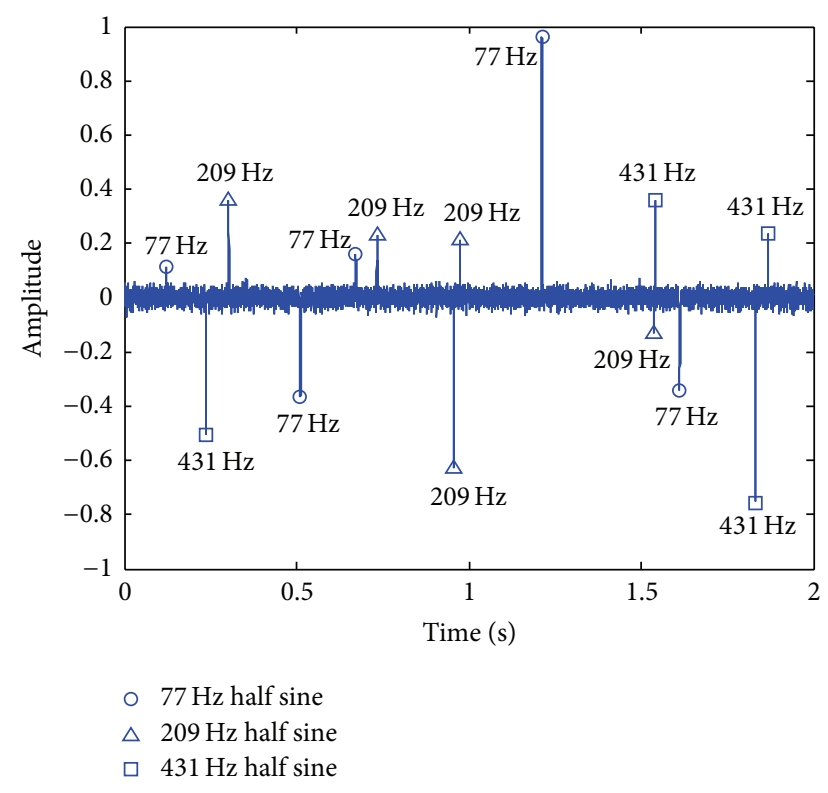

FIgURE 5: Type C impulse input with 14 half-sine waves and white noise.

14 impulses of $209 \mathrm{~Hz}$ half-sine wave with white noise for the same duration. Type $\mathrm{C}$ input contains randomly spaced 14 impulses of $77 \mathrm{~Hz}, 209 \mathrm{~Hz}$, and $431 \mathrm{~Hz}$ half-sine waves with white noise as plotted in Figure 5.

The number of the half-sine waves was 14 for each impulse input type as illustrated in Figure 5. The magnitudes, intervals, and signs of each half-sine wave were randomly decided for the experiment. Type $\mathrm{C}$ impulse input consists of 5 of $77 \mathrm{~Hz}, 5$ of $209 \mathrm{~Hz}$, and 4 of $431 \mathrm{~Hz}$ half-sine waves with white noise. The largest magnitude of the 14 impulses in Figure 5 is designed to be 1 . Each type impulse input was used as $x(t)$ signal in Figure 1 for the real-time control experiment in this study.

\section{Results and Discussions}

The real-time control experiments in the duct system against the impulse input noises as the primary sources, which are defined in Table 2 and Figure 4 in the previous section, are shown.

4.1. Control against Type A Impulse Input. As type A impulse input consists of $77 \mathrm{~Hz}$ half-sine waves mostly, the spectrogram and PSD plotted in Figures 6(a) and 6(d), respectively, show the dominant frequency components are observed around $80 \mathrm{~Hz}$ (the darkest parts) before control. It is also noted that there are some peaky components at about $350 \mathrm{~Hz}$, which was caused by the acoustic modes in the duct.

The spectrogram, PSD, and attenuation in Figures 6(b), 6(d), and 6(e), respectively, after the FxLMS control against the same input type indicate that the reduction of 17 $25 \mathrm{~dB}$ was achieved below $200 \mathrm{~Hz}$. Particularly the dominant frequency components around $80 \mathrm{~Hz}$ were suppressed substantially. The spectrogram image after the FxLMS control in Figure 6(b) becomes quite brighter than before control in Figure 6(a).

The spectrogram in Figure 6(c) after the FxNLMS control displays even brighter image than after FxLMS control in Figure 6(b). The attenuation plot in Figure 6(e) illustrates that the frequency range of noise suppression was extended to about $800 \mathrm{~Hz}$ and the amount of the reduction reached about $15 \sim 25 \mathrm{~dB}$.

It is observed that the attenuation by the FxLMS is slightly better than the FxNMLS between 50 and $100 \mathrm{~Hz}$ in Figure 6(e). In type A input, the necessary sample number of the $77 \mathrm{~Hz}$ half-sine wave is 39 because the sampling frequency was $6000 \mathrm{~Hz}$. As the fixed length of the signal $\widehat{\mathbf{x}}$ in the denominator of (2) for the FxNLMS was 100 in the control experiment, the FxNLMS could become less effective when the wavelength of the input noise is relatively long.

If the length of the signal $\widehat{\mathbf{x}}$ becomes longer, then the control effectiveness at low frequency such as $50-100 \mathrm{~Hz}$ would be better. However, the effectiveness at high frequency could become worse. Thus, this can be a problem of choosing more important frequency range for control. After intensive experiment, the length of 100 was determined to develop more effective algorithm against impulse input noises in which the frequencies of half-sine waves vary between $77 \mathrm{~Hz}$ and $431 \mathrm{~Hz}$.

4.2. Control against Type B Impulse Input. As type B impulse input comprises $209 \mathrm{~Hz}$ half-sine waves, these frequency components were cancelled extensively after either the FxLMS or the FxNLMS controls as shown in Figure 7. It is noted that the performances of both control algorithms were quite similar below about $450 \mathrm{~Hz}$ with the maximum reduction of about $30 \mathrm{~dB}$ as it can be seen from Figures $7(\mathrm{~d})$ and $7(\mathrm{e})$. The spectrogram images in Figures 7(a), 7(b), and 7 (c) demonstrate that the FxNLMS algorithm was better than that of the FxLMS.

4.3. Control against Type C Impulse Input. As type C impulse input has three half-sine waves of $77 \mathrm{~Hz}, 209 \mathrm{~Hz}$, and $431 \mathrm{~Hz}$ randomly in terms of their magnitudes and occurrence and white noise, because of the various magnitudes of the input impulses which cause the different ringing times after each impulse, as plotted in Figure 8(b), the FxLMS control cannot quickly suppress the ringing response followed by the impulse at about 1.2 seconds. It is found that the ringing response continued until the next impulse occurred.

However, the FxNLMS control suppressed the ringing response very quickly as presented in Figure 8(c). This difference of the performances of both control strategies demonstrates that the importance of the FxNLMS approach is very useful against a severe impulse input in those systems like passenger cars. In addition, it is noted that the control performance of the FxNLMS is more efficient against low frequency such as $77 \mathrm{~Hz}$ impulse input signals. The overall attenuation difference between the two algorithms in Figure $8(\mathrm{e})$ is about $5 \sim 10 \mathrm{~dB}$ at the frequency range of about $70 \sim 200 \mathrm{~Hz}$. The spectrogram images in this result clarify the performance differences in the suppression of the mixed impulse input (type C). 


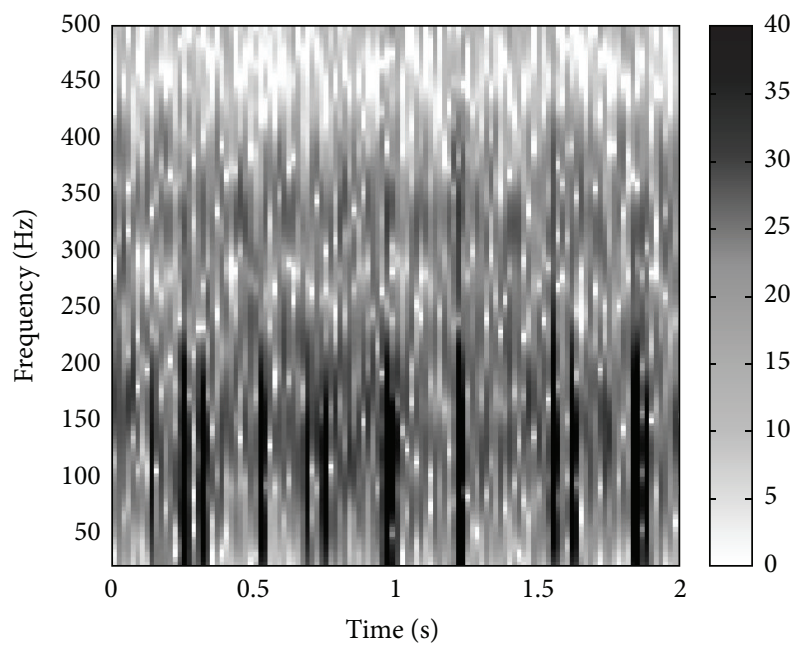

(a)

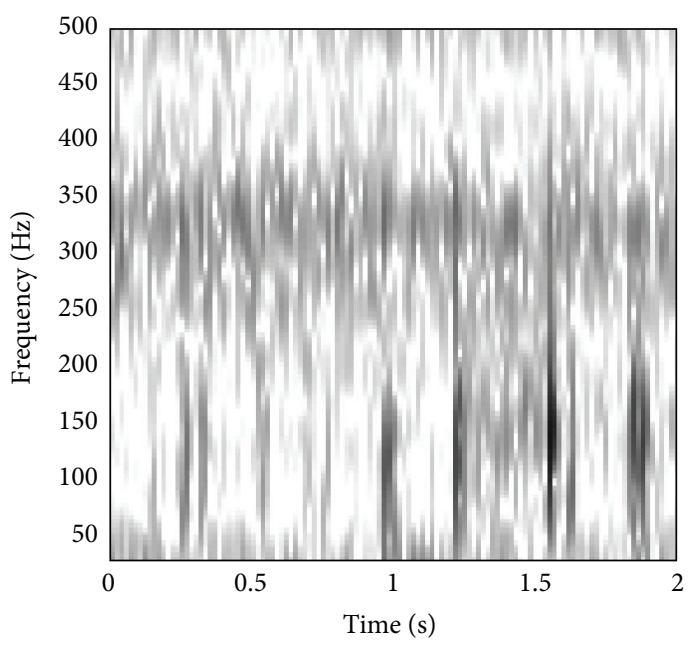

(b)

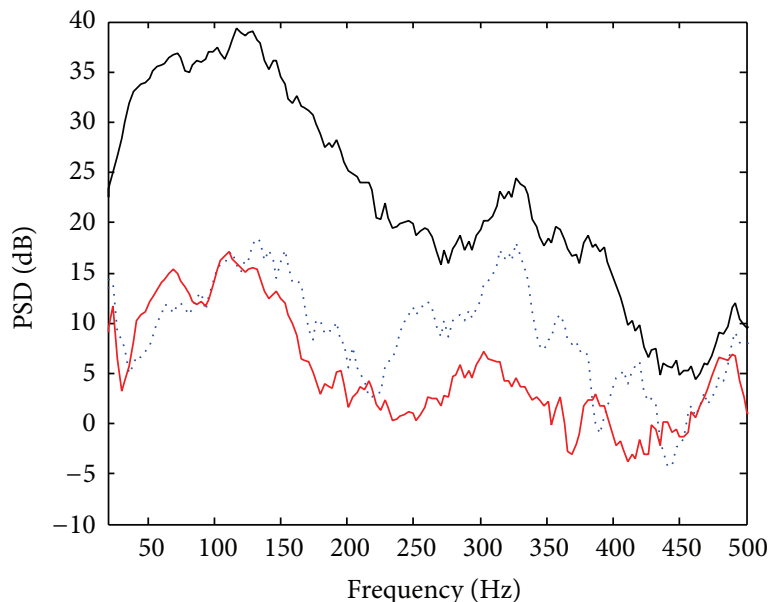

- Before control After FxLMS control _ After FxNLMS control

(d)

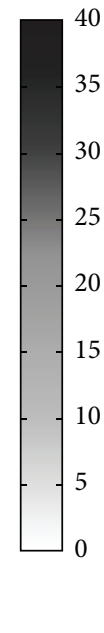

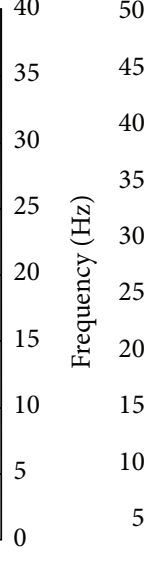

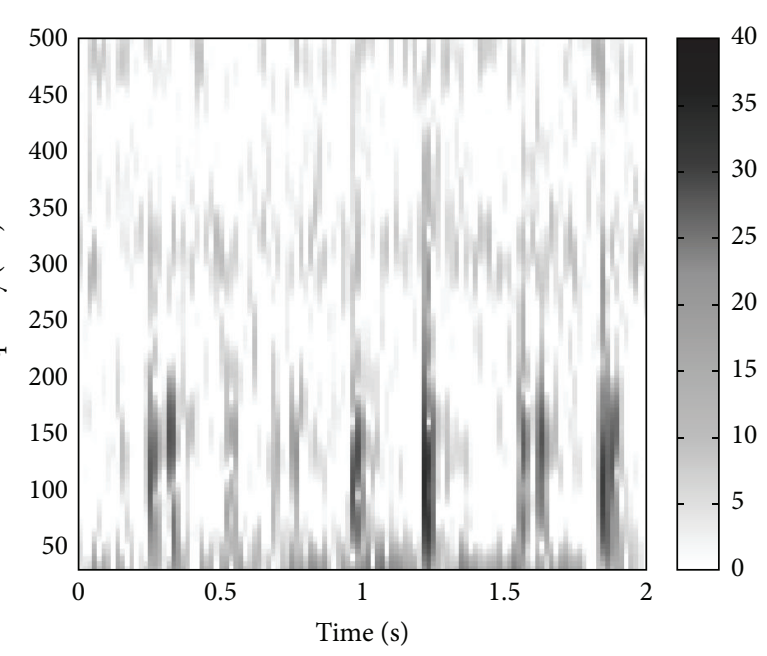

(c)

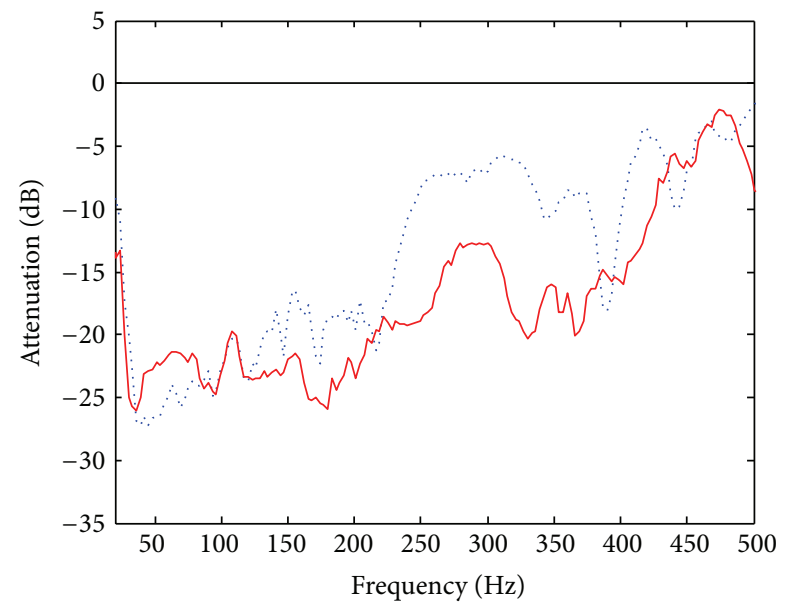

…. After FxLMS control
— After FxNLMS control

(e)

FIGURE 6: Real-time control result against type A impulse input. (a) Before control, (b) after FxLMS control, (c) after FxNLMS control, (d) PSD, and (e) attenuation. 


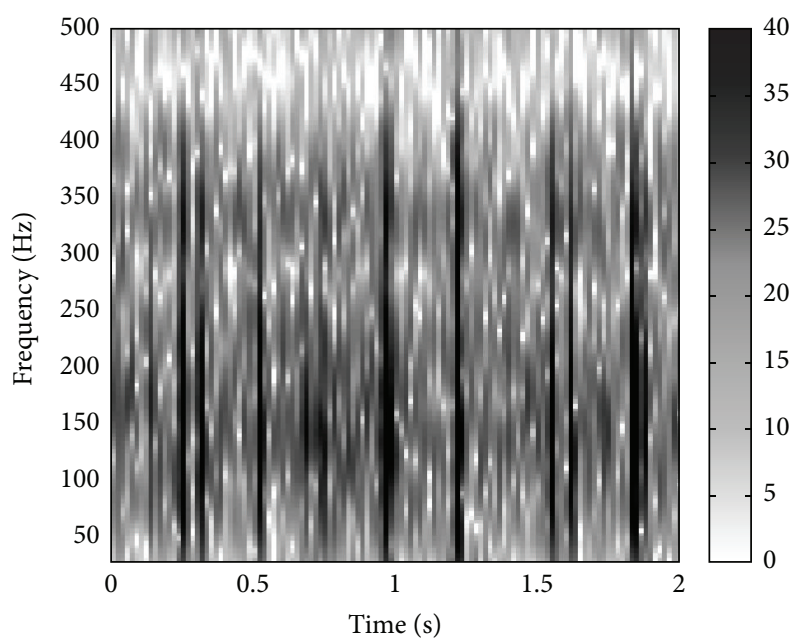

(a)

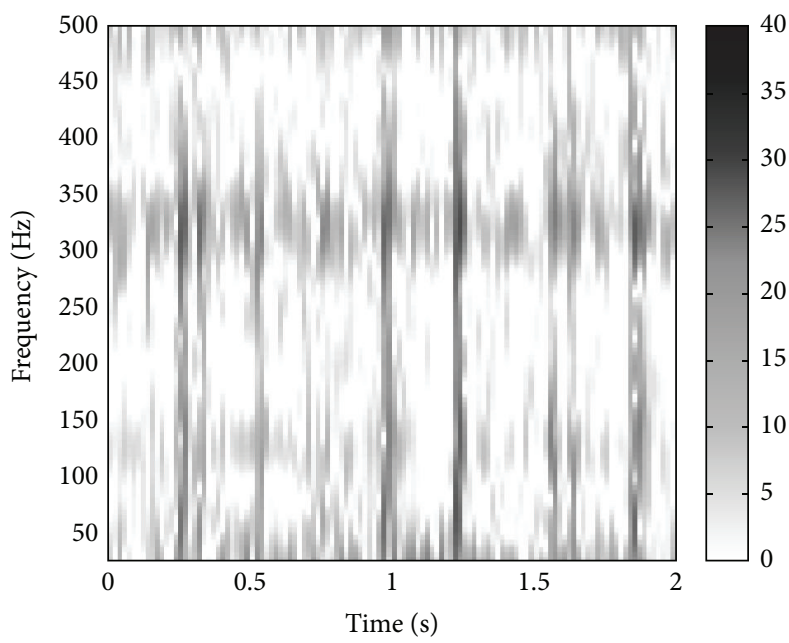

(b)

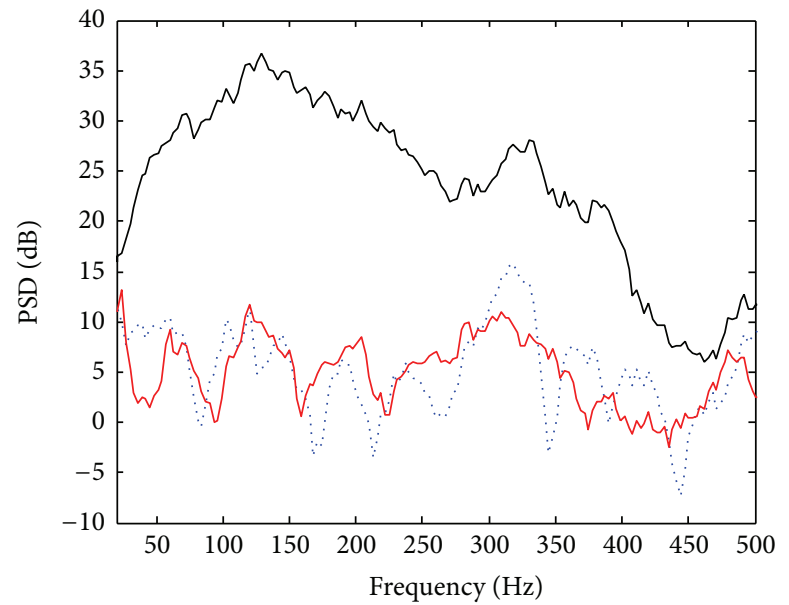

$\begin{array}{ll}\text { _.... } & \text { Before control } \\ \text {... After FxLMS control } \\ \text { After FxNLMS control }\end{array}$

(d)

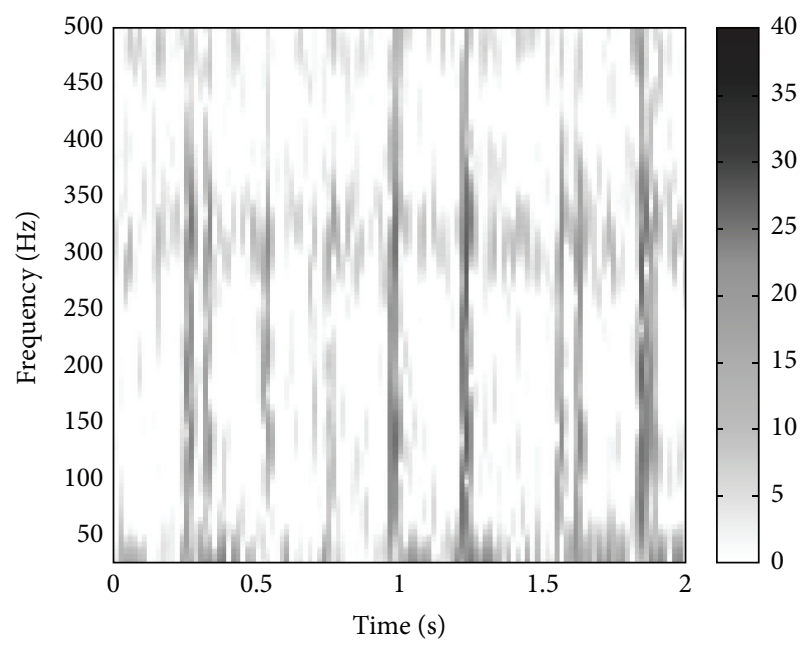

(c)

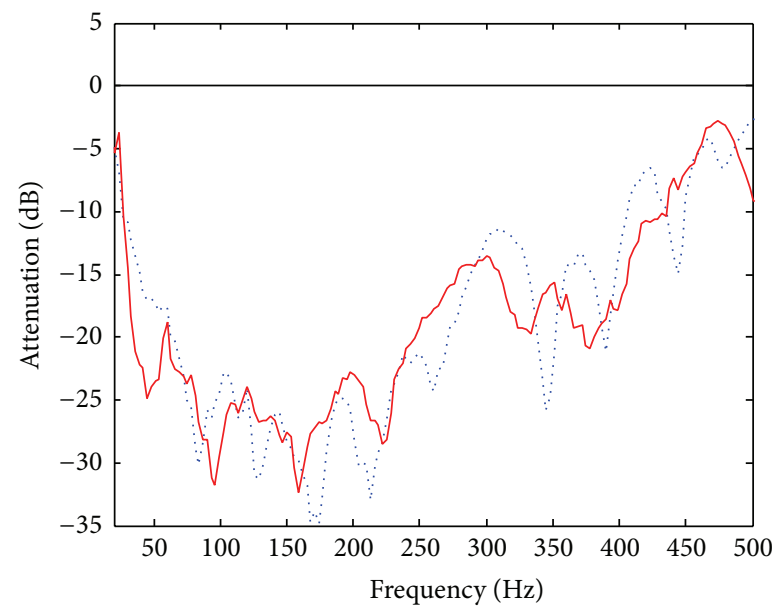

...... After FxLMS control

— After FxNLMS control

(e)

FiguRE 7: Real-time control result against type B impulse input. (a) Before control, (b) after FxLMS control, (c) after FxNLMS control, (d) PSD, and (e) attenuation. 


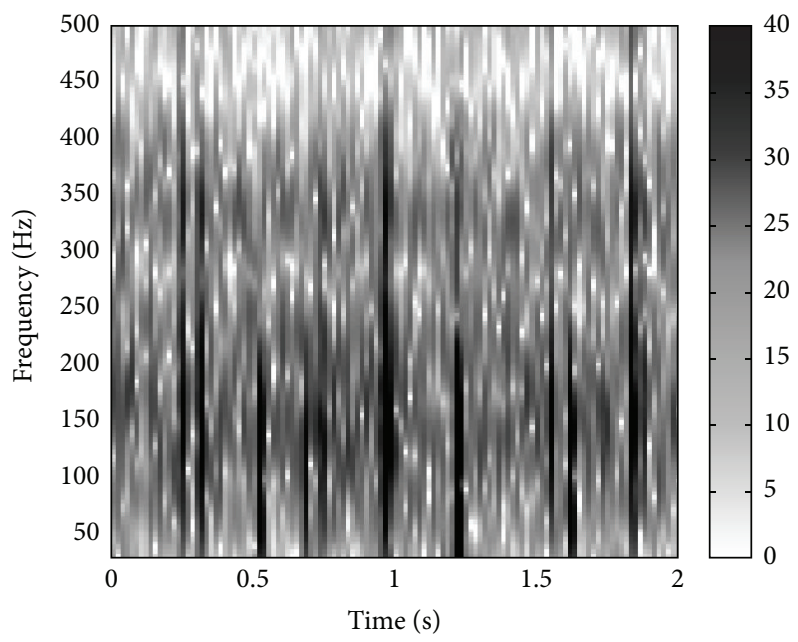

(a)

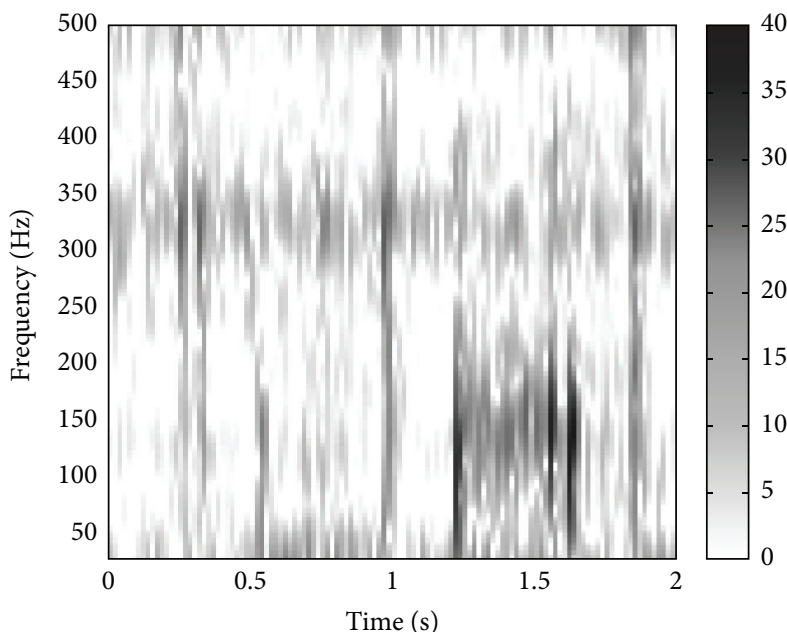

(b)

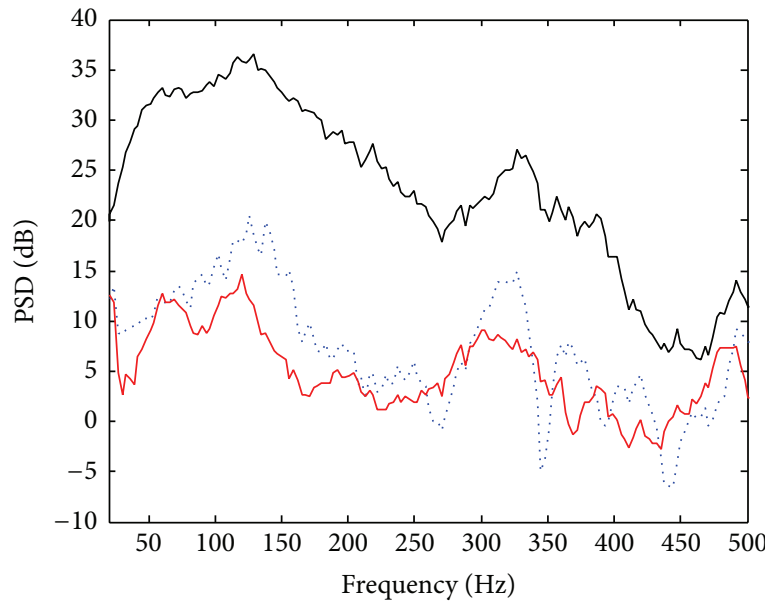

_ Before control After FxLMS control — After FxNLMS control

(d)

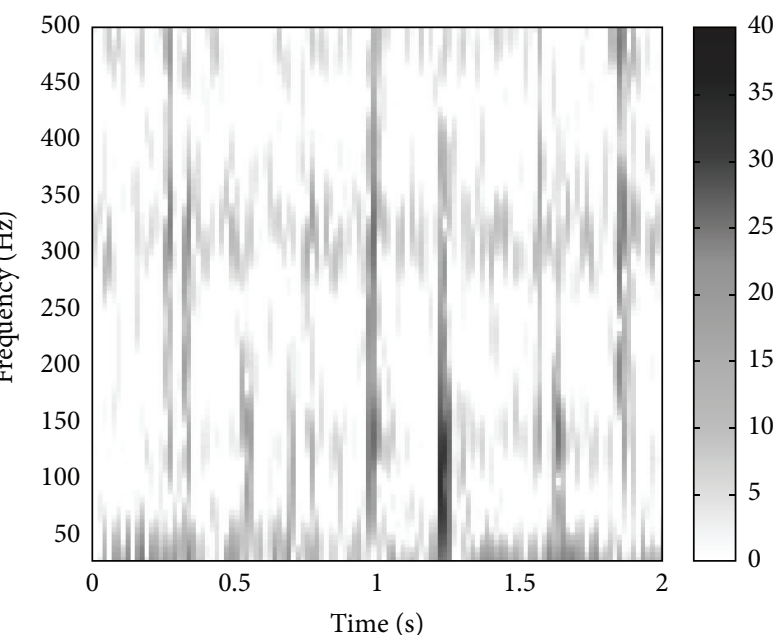

(c)

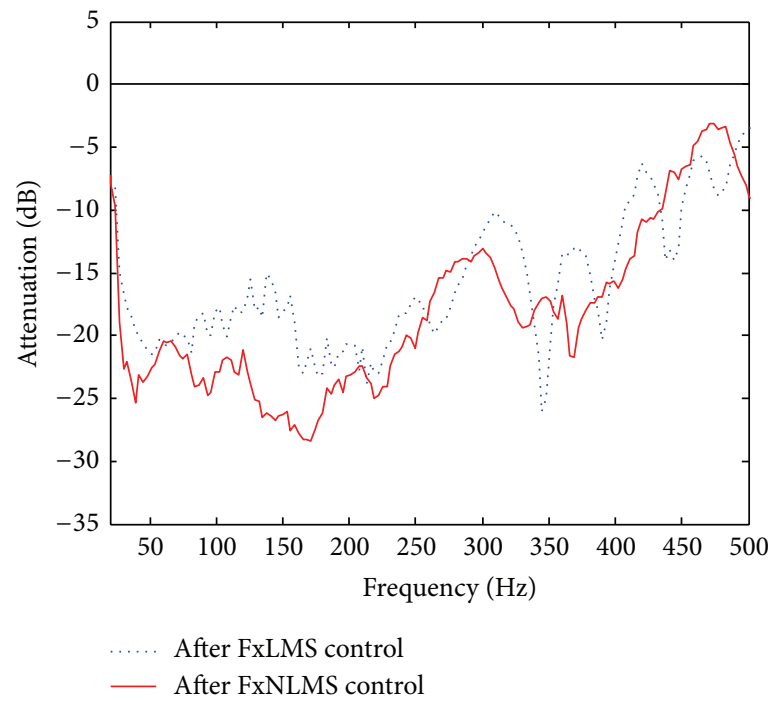

(e)

FIGURE 8: Real-time control result against type C impulse input. (a) Before control, (b) after FxLMS control, (c) after FxNLMS control, (d) PSD, and (e) attenuation. 
TABLE 3: Overall attenuation in 20 500 Hz.

\begin{tabular}{lcc}
\hline \multirow{2}{*}{ Impulse input type } & \multicolumn{2}{c}{ Overall attenuation } \\
& FxLMS & FxNLMS \\
\hline Type A & $-13.44 \mathrm{~dB}$ & $-16.97 \mathrm{~dB}$ \\
Type B & $-18.03 \mathrm{~dB}$ & $-18.24 \mathrm{~dB}$ \\
Type C & $-15.77 \mathrm{~dB}$ & $-17.80 \mathrm{~dB}$ \\
\hline
\end{tabular}

Table 3 presents the average attenuations in $\mathrm{dB}$ against the three impulse input types A, B, or C. As plotted in Figures 6,7 , and 8 , the differences in the three spectrogram images are caused by the impulse inputs that are different. The FxNLMS algorithm embedded in the real-time digital controller responds automatically with adaptive algorithms of the FxLMS and the FXNLMS against the input noises. Thus, the spectrogram images must be different because the two algorithms are different. Since the inputs were impulses, the FxNLMS showed better performance than the FxLMS in most of the frequency range as summarized in Table 3. The FxNLMS algorithm performed $-16.97 \mathrm{~dB},-18.24 \mathrm{~dB}$, and $-17.80 \mathrm{~dB}$ against the impulse input types $\mathrm{A}, \mathrm{B}$, and $\mathrm{C}$, respectively. By the way, the FxLMS algorithm accomplished $-13.44 \mathrm{~dB},-18.03 \mathrm{~dB}$, and $-15.77 \mathrm{~dB}$ in the same order. This indicates that the FxNLMS algorithm achieved about $3.5 \mathrm{~dB}$, $0.2 \mathrm{~dB}$, and $2.0 \mathrm{~dB}$ more attenuation than the FxLMS.

Comparing the performances of the two algorithms against especially type $\mathrm{C}$ impulses input which is expected as more practical, it is noted the FxNLMS provides better stability for suppressing the resonances of the duct system. The two largest resonances at about $125 \mathrm{~Hz}$ and $325 \mathrm{~Hz}$ before control as plotted in Figure 8(d) were suppressed more about $7 \sim 15 \mathrm{~dB}$ by the FxNLMS than the FxLMS.

The frequency variation of the impulse input gives important effect to the stability and the performance of the FxNLMS algorithm, since the length of the signal $\widehat{\mathbf{x}}$ in the denominator of (2) for the FxNLMS is very much related to the input halfsine wave frequency. Thus, if it is necessary to minimize a higher frequency impulse input, then a shorter length of the signal $\widehat{\mathbf{x}}$ is required. Against low frequency impulses noises, a longer length is better in general. If the variation of the frequency of a half-sine impulse is small, the effect will not be big.

\section{Conclusions}

This study presents spectrogram images analysis of error signals in an active control system against the impulse input signals in a one-dimensional duct. For the improvement of spectrogram images, a new normalization factor $\alpha_{N C}$ in the update equation was implemented for the real-time control algorithm.

The frequency variation of the impulse input gives important effect to the stability and the performance of the FxNLMS algorithm, since the length of the signal $\widehat{\mathbf{x}}$ in the denominator for the FxNLMS is very much related to the input half-sine wave frequency.

Control results with the new algorithm showed stable and excellent responses compared to those before control.
As the three impulse input signals types with white noise were generated independently, the control algorithm attenuates the noises from about $-17 \mathrm{~dB}$ to $-18 \mathrm{~dB}$ with the new normalization factor in the real-time control. On the other hand, only about $-13 \mathrm{~dB}$ to $-16 \mathrm{~dB}$ were reduced with the FxLMS algorithm. The improved algorithm also worked with a nice stability and performance against 3 different types of the impulse input signals. The results in this study can be used for practical active noise control systems.

\section{Conflict of Interests}

The authors declare that there is no conflict of interests regarding the publication of this paper.

\section{Acknowledgment}

This research was supported by a grant (code 15CCTIB062410-04-000000) from Advanced Water Management Research Program funded by Ministry of Land, Infrastructure and Transport of Korean government.

\section{References}

[1] G. Jeon, S. Kang, and Y.-S. Lee, "Noise level estimation for image processing," in Convergence and Hybrid Information Technology, vol. 7425 of Lecture Notes in Computer Science, pp. 350-356, 2012.

[2] Y.-S. Lee, P. Gardonio, and S. J. Elliott, "Coupling analysis of a matched piezoelectric sensor and actuator pair for vibration control of a smart beam," Journal of the Acoustical Society of America, vol. 111, no. 6, pp. 2715-2726, 2002.

[3] G. Jeon, M. Anisetti, V. Bellandi, E. Damiani, and J. Jeong, "Fuzzy weighted approach to improve visual quality of edgebased filtering," IEEE Transactions on Consumer Electronics, vol. 53, no. 4, pp. 1661-1667, 2007.

[4] P. Gardonio, Y.-S. Lee, S. J. Elliott, and S. Debost, "Analysis and measurement of a matched volume velocity sensor and uniform force actuator for active structural acoustic control," The Journal of the Acoustical Society of America, vol. 110, no. 6, pp. 3025-3031, 2001.

[5] D. Bismor, "LMS algorithm step size adjustment for fast convergence," Archives of Acoustics, vol. 37, no. 1, pp. 31-40, 2012.

[6] S. O. Haykin, Adaptive Filter Theory, Prentice-Hall, 5th edition, 2013.

[7] S. J. Elliott, Signal Processing for Active Control, Academic Press, 2001.

[8] Y.-S. Lee and S. J. Elliott, "Active position control of a flexible smart beam using internal model control," Journal of Sound and Vibration, vol. 242, no. 5, pp. 767-791, 2001.

[9] K. Shin, S. Pyo, and Y.-S. Lee, "Simple feed-forward active control method for suppressing the shock response of a flexible cantilever beam," Smart Materials and Structures, vol. 18, no. 2, Article ID 027001, 6 pages, 2009.

[10] G. Jeon, S. Kang, and Y.-S. Lee, "A filter-based format conversion approach," Communications in Computer and Information Science, vol. 310, pp. 559-565, 2012.

[11] G. Jeon, S. Kang, and Y.-S. Lee, "Weight assignment method for interpolation," Communications in Computer and Information Service, vol. 342, pp. 272-278, 2012. 
[12] R. M. Reddy, I. M. S. Panahi, and R. Briggs, "Hybrid FxRLSFxNLMS adaptive algorithm for active noise control in fMRI application," IEEE Transactions on Control Systems Technology, vol. 19, no. 2, pp. 474-480, 2011.

[13] K. Sivadasan and N. K. Narayanan, "ANC system for noisy speech," Signal and Image Processing International Journal, vol. 3, no. 3, pp. 93-101, 2012.

[14] K. K. Das and J. K. Satapathy, "Frequency-domain block filtered-x NLMS algorithm for multichannel ANC," in Proceedings of the 1st International Conference on Emerging Trends in Engineering and Technology (ICETET '08), pp. 1293-1297, IEEE, Nagpur, India, July 2008.

[15] Y. Kajikawa and R. Hirayama, "Feedback active noise control system combining linear prediction filter," in Proceedings of the European Signal Processing Conference, pp. 31-35, August 2010. 


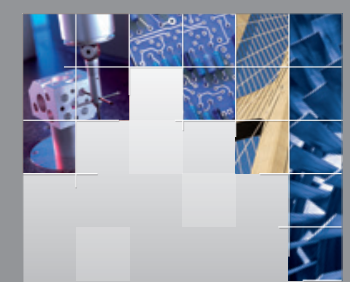

\section{Enfincering}
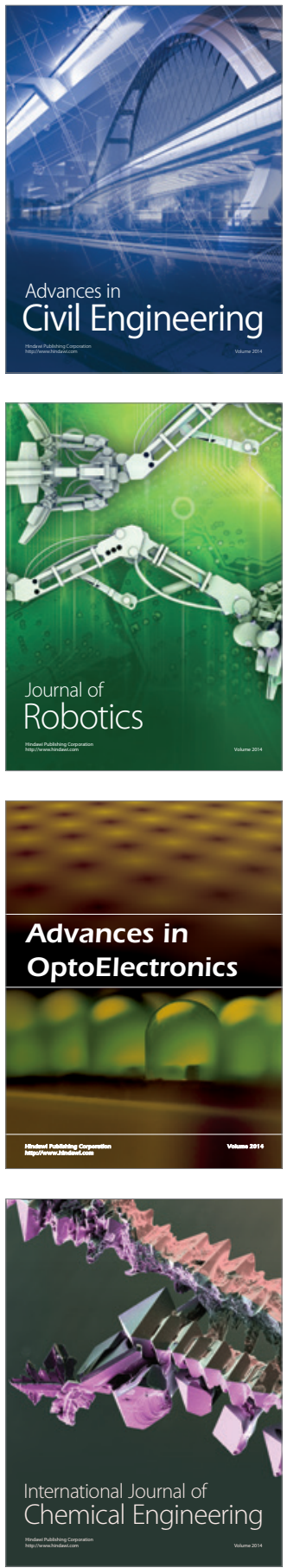

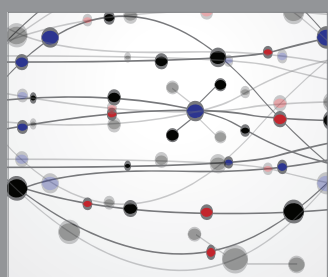

The Scientific World Journal

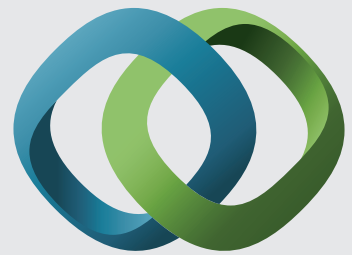

\section{Hindawi}

Submit your manuscripts at

http://www.hindawi.com
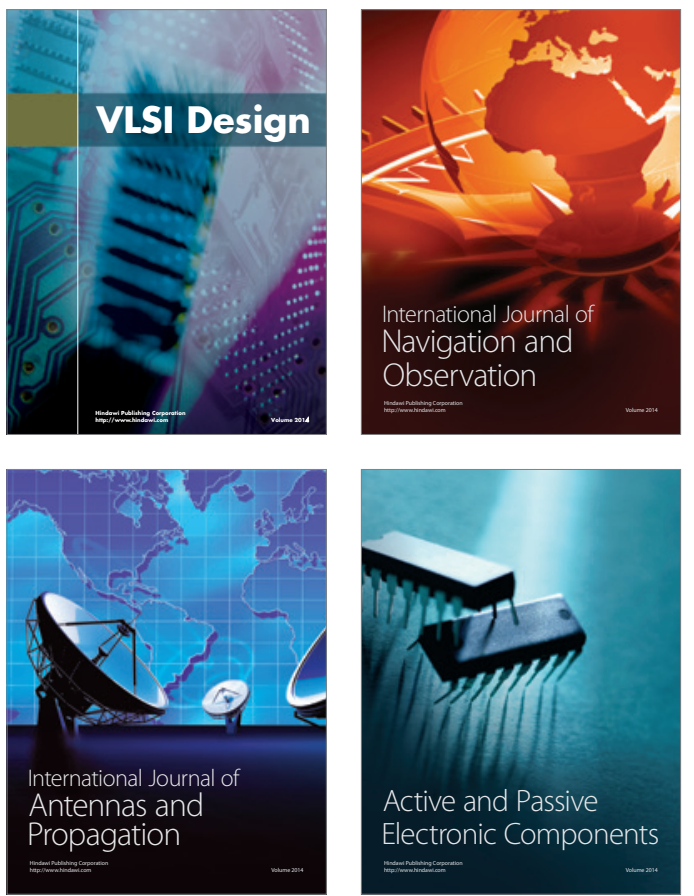
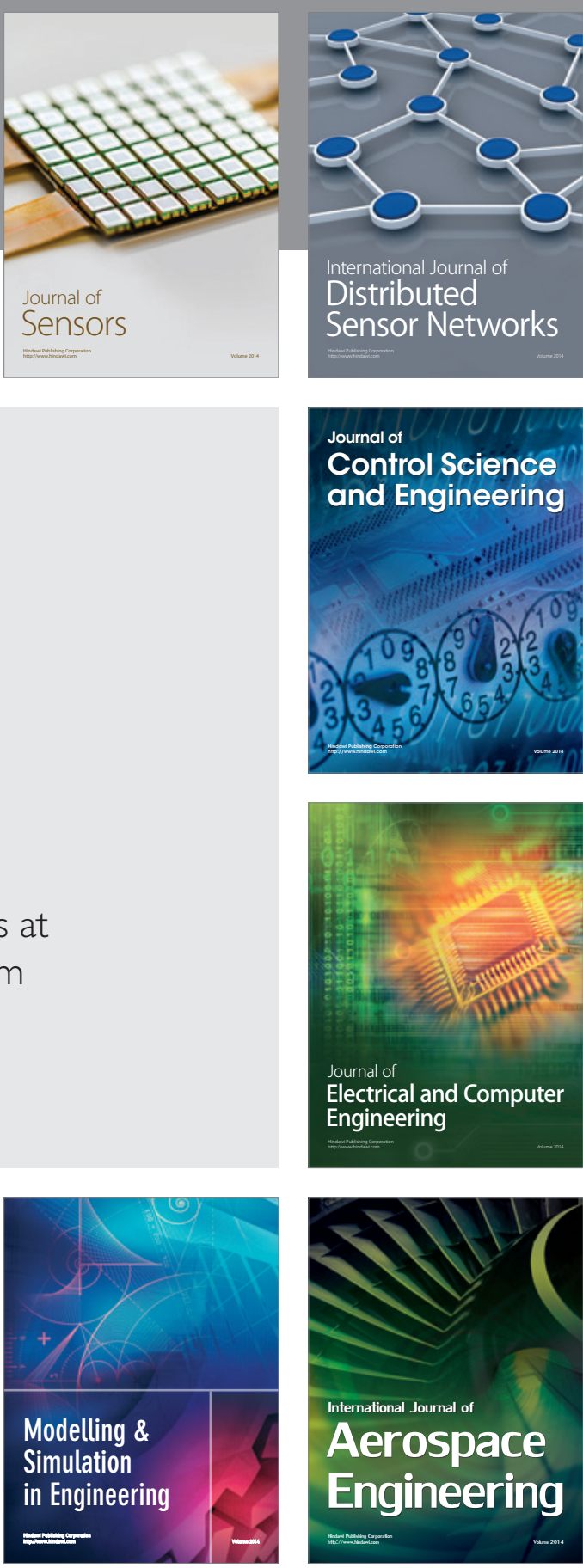

International Journal of

Distributed

Sensor Networks

Journal of

Control Science

and Engineering
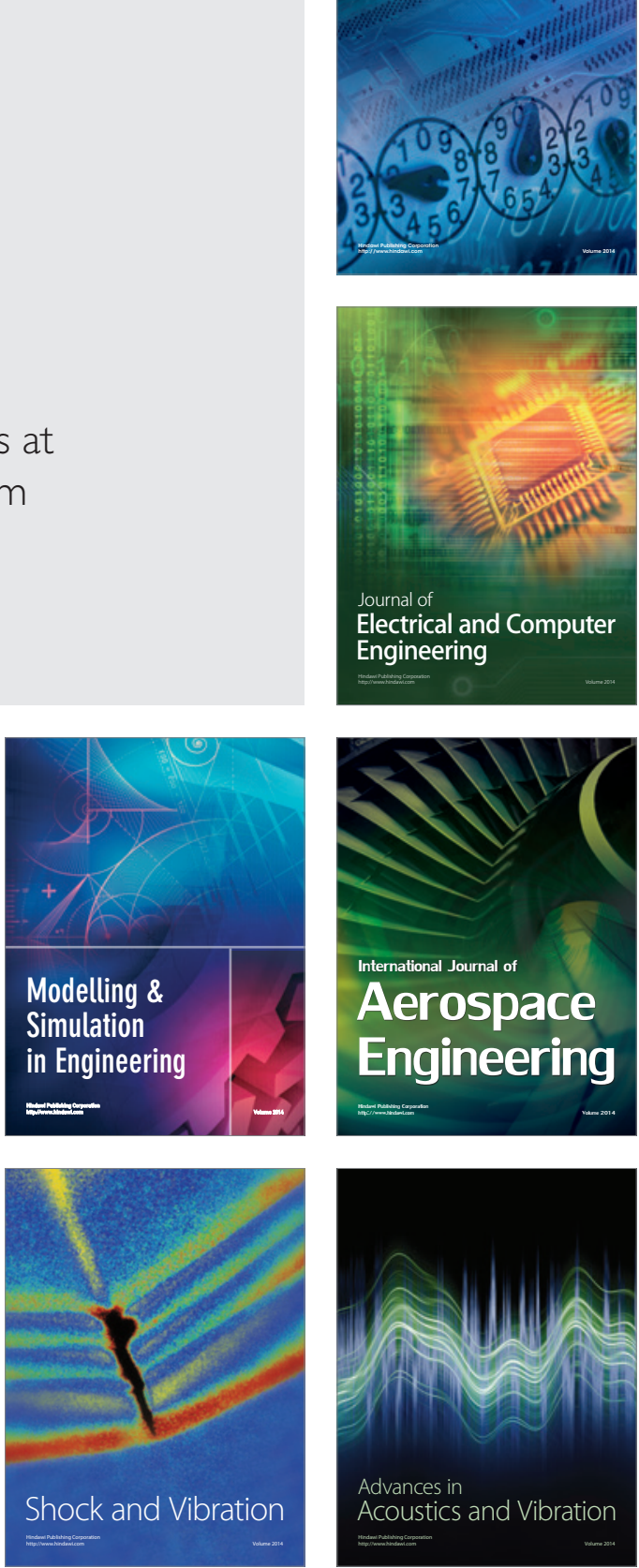